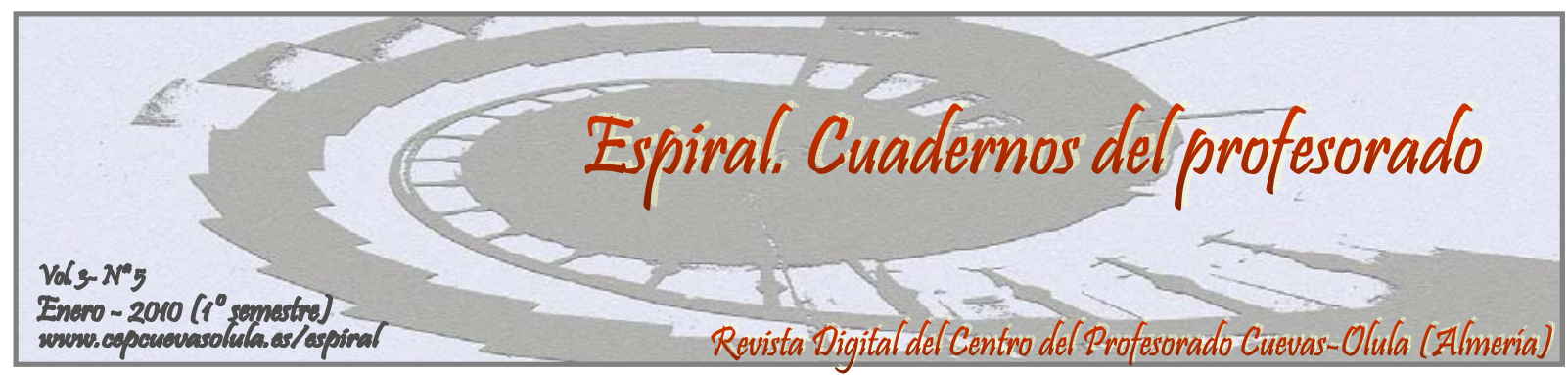

\title{
LA IMPORTANCIA DE LA SALUD LABORAL DOCENTE: ESTUDIO DE UN CASO CONCRETO
}

\author{
THE IMPORTANCE OF TEACHERS' HEALTH AT WORK: STUDY OF A \\ PARTICULAR CASE
}

\section{Luz María Montiel Hijano}

\author{
Licenciada en Filología Inglesa, Profesora de Escuela Oficial de Idiomas
}

RESUMEN: Este artículo trata el tema de la salud laboral docente en los centros educativos, se analizan los problemas de salud mental y física que más atañen al profesorado y se proponen métodos de prevención y posibles soluciones que el personal docente y no docente de los centros pueden llevar a la práctica. El método fundamental que se ha llevado a cabo es recabar información acerca de la salud laboral docente a través de los estudios y conclusiones de los profesionales médicos, psicólogos y el propio personal docente-, reflexionar sobre ella y tratar de solventar los problemas de salud que podamos desarrollar en nuestros centros de trabajo. Además de analizar estos temas, también se prestará una especial atención a los resultados obtenidos en nuestro centro después de haber entrevistado y encuestado al personal docente y no docente. A modo de conclusión podemos afirmar que es el sistema educativo quien debe promover la instauración de una cultura de prevención de riesgos laborales en nuestra sociedad. Para ello, se debe concienciar al personal de los centros educativos de la importancia que tiene la salud en nuestro trabajo y se les debe animar a tomar las medidas oportunas para cambiar aquellos aspectos que no funcionan en nuestros estilos de vida.

Palabras clave: salud laboral, profesorado, medidas preventivas, enfermedades.

ABSTRACT: This article deals with the topic of teachers' health at work in educational centres. It analyses the mental and physical health problems which concern the teachers most and it also suggests ways of prevention and possible solutions which can be put into practise by the school staff. The basic method which has been carried out is that of gathering information about teachers' health at work by means of the studies and conclusions of professionals -doctors, psychologists and the teaching staff themselves-, reflecting on it and trying to solve the health problems which can be developed in our workplaces. Apart from analysing these topics, special attention will also be paid to the results obtained from the interviews and surveys which have been achieved by the school staff in our centre. In conclusion, it can be stated that it is the educational system the one which must promote the implementation of an occupational risk prevention culture in our society. To that end, the staff from our educational centres must be aware of the importance of health at work. They must also be encouraged to adopt appropriate measures in order to change those aspects which are not working in our lifestyles.

Key words: health at work, teachers, preventive measures, illnesses. 
Montiel, L.M. (2010). La importancia de la salud laboral docente: estudio de caso. Espiral. Cuadernos del Profesorado [en línea], 3(5), 11-29. Disponible en: http://www.cepcuevasolula.es/espiral.

Fecha de recepción: 14/10/2009

Fecha de aceptación: 21/12/2009

\section{1.- INTRODUCCIÓN.}

Para comenzar a describir este artículo acerca de la Salud Laboral Docente, sería conveniente partir del Acuerdo de 19 de septiembre de 2006, del Consejo de Gobierno, por el que se aprueba el I Plan Andaluz de Salud Laboral y Prevención de Riesgos Laborales del personal docente de los centros públicos dependientes de la Consejería de Educación (BOJA $\mathrm{n}^{\circ} 196$ de 9 de octubre de 2006). Según este Plan, el Gobierno de la Junta de Andalucía tiene como objetivo el fomento de una cultura auténtica de la prevención de los riesgos derivados de las condiciones de trabajo, la promoción de la salud laboral, la reducción de los accidentes de trabajo y de las enfermedades relacionadas con el puesto de trabajo, así como la mejora de las condiciones de trabajo en Andalucía.

Tal y como se recoge en el Protocolo de colaboración de este acuerdo, se contempla la realización de algunas acciones, tales como la organización de actividades formativas en materia de prevención de riesgos laborales destinadas al profesorado, publicaciones dirigidas a este colectivo y asesoramiento a través de talleres.

La definición más conocida de la salud es de la Organización Mundial de la Salud (OMS): "La salud es el estado completo de bienestar físico, mental y social y no solamente la ausencia de enfermedades". No solamente significa verse libre de dolores o enfermedades sino también la libertad de desarrollar y mantener sus capacidades funcionales físicas, psíquicas y sociales.

Cuando utilizamos el término de salud laboral nos referimos a aquellos estudios o acciones que tienen como finalidad conocer la importancia del trabajo en las alteraciones de la salud en una población, así como las medidas preventivas que se pueden realizar en el marco laboral (López López-Menchero, 2009).

Creemos que es esencial desarrollar hábitos y costumbres saludables, crear entornos adecuados y valorarlos como uno de los aspectos
Enviar correspondencia a: zulmh@yahoo.es

básicos de la calidad de vida, corregir las deficiencias ambientales y rechazar aquellas pautas de comportamiento que conducen a un malestar físico, mental y emocional. Por ello, en este artículo hemos partido por estudiar el trabajo que desarrolla el personal en nuestro centro y las necesidades que tenemos, hemos elaborado encuestas anónimas para detectar los problemas de salud más habituales entre el personal docente y no docente, y también hemos planteado posibles soluciones para mejorar nuestra salud laboral, el cual es nuestro objetivo meta.

\section{2.- PREVENCIÓN DE RIESGOS LABORALES.}

La Ley 31/1995, de 8 de noviembre, de Prevención de Riesgos Laborales (BOE $n^{\circ}$ 269, de 10 de noviembre de 1995) traspone al derecho español las normativas y directivas europeas relativas a la aplicación de las medidas para promover la mejora de la salud y la seguridad de los trabajadores y trabajadoras, así como el marco jurídico en el que se integra la política de prevención comunitaria.

En el artículo 14 de la presente ley se trata el derecho a la protección frente a los riesgos laborales y se expone lo siguiente:

- Los trabajadores y trabajadoras tienen derecho a una protección eficaz en materia de seguridad y salud en el trabajo. El citado derecho supone la existencia de un correlativo deber del empresario o empresaria de protección de los trabajadores y trabajadoras frente a los riesgos laborales. Este deber de protección constituye, igualmente, un deber de las Admones. públicas respecto del personal a su servicio.

- Los derechos de información, consulta y participación, formación en materia preventiva, paralización de la actividad en caso de riesgo grave e inminente y vigilancia de su estado de salud, en los términos previstos en la presente Ley, forman parte del derecho de los trabajadores y trabajadoras a una protección eficaz en materia de seguridad y salud en el trabajo. 
- En cumplimiento del deber de protección, el empresario o empresaria deberá garantizar la seguridad y la salud de los trabajadores y trabajadoras a su servicio en todos los aspectos relacionados con el trabajo. A estos efectos, en el marco de sus responsabilidades, el empresario o empresaria realizará la prevención de los riesgos laborales mediante la integración de la actividad preventiva en la empresa y la adopción de cuantas medidas sean necesarias para la protección de la seguridad y la salud de los trabajadores y trabajadoras, evaluación de riesgos, información, consulta y participación y formación de los trabajadores y trabajadoras, actuación en casos de emergencia y de riesgo grave e inminente, vigilancia de la salud, y mediante la constitución de una organización y de los medios necesarios establecidos en esta ley.

- El empresario o empresaria desarrollará una acción permanente de seguimiento de la actividad preventiva con el fin de perfeccionar de manera continua las actividades de identificación, evaluación y control de los riesgos que no se hayan podido evitar y los niveles de protección existentes y dispondrá lo necesario para la adaptación de las medidas de prevención señaladas en el párrafo anterior a las modificaciones que puedan experimentar las circunstancias que incidan en la realización del trabajo. El empresario o empresaria deberá cumplir las obligaciones establecidas en la normativa sobre prevención de riesgos laborales.

- Las obligaciones de los trabajadores y trabajadoras establecidas en esta Ley, la atribución de funciones en materia de protección y prevención a trabajadores y trabajadoras o servicios de la empresa y el recurso al concierto con entidades especializadas para el desarrollo de actividades de prevención complementarán las acciones del empresario o empresaria, sin que por ello le eximan del cumplimiento de su deber en esta materia, sin perjuicio de las acciones que pueda ejercitar, en su caso, contra cualquier otra persona. El coste de las medidas relativas a la seguridad y la salud en el trabajo no deberá recaer en modo alguno sobre los trabajadores y trabajadoras.
En el artículo 15 de la presente ley se tratan los principios de la acción preventiva, que es el factor de mayor importancia en la prevención de los riesgos laborales, y se expone lo siguiente:

- El empresario o empresaria aplicará las medidas que integran el deber general de prevención previsto en el artículo anterior, con arreglo a los siguientes principios generales:

a) Evitar los riesgos.

b) Evaluar los riesgos que no se puedan evitar.

c) Combatir los riesgos en su origen.

d) Adaptar el trabajo a la persona, en particular en lo que respecta a la concepción de los puestos de trabajo, así como a la elección de los equipos y los métodos de trabajo y de producción, con miras, en particular, a atenuar el trabajo monótono y repetitivo y a reducir los efectos del mismo en la salud.

e) Tener en cuenta la evolución de la técnica.

f) Sustituir lo peligroso por lo que entrañe poco o ningún peligro.

g) Planificar la prevención, buscando un conjunto coherente que integre en ella la técnica, la organización del trabajo, las condiciones de trabajo, las relaciones sociales y la influencia de los factores ambientales en el trabajo.

h) Adoptar medidas que antepongan la protección colectiva a la individual.

i) Dar las debidas instrucciones a los trabajadores y trabajadoras.

- El empresario o empresaria tomará en consideración las capacidades profesionales de los trabajadores y trabajadoras en materia de seguridad y de salud en el momento de encomendarles las tareas.

- El empresario o empresaria adoptará las medidas necesarias a fin de garantizar que sólo los trabajadores y trabajadoras que hayan recibido información suficiente y adecuada puedan acceder a las zonas de riesgo grave y específico.

- La efectividad de las medidas preventivas deberá prever las distracciones o imprudencias no temerarias que pudiera cometer el trabajador o trabajadora. Para su adop- 
ción se tendrán en cuenta los riesgos adicionales que pudieran implicar determinadas medidas preventivas, las cuales sólo podrán adoptarse cuando la magnitud de dichos riesgos sea sustancialmente inferior a la de los que se pretende controlar y no existan alternativas más seguras.

- Podrán concertar operaciones de seguro que tengan como fin garantizar como ámbito de cobertura la previsión de riesgos derivados del trabajo, la empresa respecto de sus trabajadores y trabajadoras, los trabajadores y trabajadoras autónomos/as respecto a ellos/as mismos/as y las sociedades cooperativas respecto a sus socios y socias cuya actividad consista en la prestación de su trabajo personal.

Por su parte, el Decreto 117/2000, de 11 de abril, por el que se crean los Servicios de Prevención de Riesgos Laborales para el personal al servicio de la Administración de la Junta de Andalucía (BOJA no 45, de 15 de abril de 2000), contempla que serán los Centros de Prevención de Riesgos Laborales, dependientes de la Consejería de Empleo, los órganos encargados de llevar a cabo dichas funciones. Mediante el citado decreto, se constituye un Servicio de Prevención en cada provincia con competencias en todos y cada uno de los centros de trabajo incluidos dentro de la organización de la Administración autonómica, así como sobre el personal que preste sus servicios en dicho ámbito territorial y funcional.

\section{3.- PROBLEMAS DE SALUD LABORAL DOCENTE.}

\section{Salud mental.}

Los cambios que se están produciendo en la sociedad actual están dando lugar a que surjan problemas que afectan a la labor docente, llegando a incidir en su salud: la indisciplina del alumnado (López Fernández, 2000), la delegación de responsabilidades educativas propias de la familia al docente, el cambio de los valores y las relaciones sociales, la escasa valoración del profesorado, la diversidad de alumnado, el trasvase de responsabilidades al docente por falta de personal especializado, etc.

Todo ello ha dado lugar al hecho de que se produzca un cambio en las causas de las bajas laborales entre los profesionales de la educación, pues cada vez son más los docentes que al desarrollar su labor padecen dolencias de tipo psicosocial que han desplazado a enfermedades tradicionales en este colectivo: problemas en la voz, esquelético-musculares, infecciosos, etc.

Las condiciones laborales de los profesores y profesoras centran en la actualidad la atención de la comunidad educativa y de los profesionales de la salud mental. Incluso en la sociedad hay cierta inquietud por el estado psicológico de los docentes, en ocasiones provocada por algunos lamentables sucesos recogidos por los medios de comunicación.

En el ámbito escolar se han extendido los problemas de estrés, ansiedad y depresión que se traducen frecuentemente en bajas laborales. Estos trastornos no son exclusivos del profesorado. La prisa, la competitividad y los cambios vertiginosos amenazan el equilibrio de muchos trabajadores y trabajadoras.

En primer lugar, hay que decir que el profesorado constituye un grupo muy heterogéneo. Con todo, sí hay algunas notas comunes a todo el colectivo, entre las que salta a la vista el trato con personas. Aún cuando la relación con los alumnos y alumnas es uno de los aspectos potencialmente más gratificantes, lo cierto es que en un número significativo de casos esta comunicación está presidida por la tensión, ya sea por indisciplina de los escolares o porque el docente carece de habilidades sociales (Martínez-Otero, 2003).

Por otra parte, la actividad educativa está saturada de responsabilidades (López LópezMenchero, 2009). Es frecuente que el profesor se mantenga hipervigilante durante varias horas al día y que asuma funciones policiales y parentales que le abocan al agotamiento profesional. La sobrecarga de tareas unida a las frustraciones, insatisfacciones y a la falta de entendimiento con otros miembros de la comunidad educativa puede desencadenar en el profesorado alteraciones como: fatiga, descenso de la concentración y del rendimiento, ansiedad, insomnio, trastornos digestivos, etc. (Martínez-Otero, 2003).

En ocasiones, la presión ocupacional golpea al profesor o profesora con tanta fuerza que desequilibra su organismo y consume su energía. Este desgaste nos lleva a pensar en el "síndrome de agotamiento profesional" ("síndrome del quemado", en inglés burnout) que se manifiesta en el cansancio psicofísico y en el abatimiento, como expone el Gabinete de Seguridad y Salud Laboral (G.S.S.L.D.) (2009). Los profesores y profesoras que padecen este problema pueden 
experimentar cambios a nivel cognitivo (dificultad para mantener la atención, ideas de que son atacados por compañeros y compañeras o alumnado, etc.), en el plano emocional (tristeza profunda, irritabilidad...) y en la conducta (consumo de tóxicos, abandono del trabajo, etc.).

Fuentes de malestar docente:

A veces se da malestar en el profesorado aunque no desemboque en agotamiento profesional propiamente dicho. Algunas de las fuentes más comunes de esta insatisfacción son (Martínez-Otero, 2003):

- Inquietud e incertidumbre ante el futuro legislativo: El cambiante marco normativo que en materia educativa se ha producido en los últimos años genera desasosiego en un considerable sector del profesorado, al tiempo que supone un sobreesfuerzo por la adopción de nuevas estrategias de adaptación. Además tenemos que nombrar en este caso a todo el funcionario interino que también sufre de ansiedad y angustia con cada normativa nueva y con cada destino provisional, ya que no tiene seguridad en su trabajo.

- Prestigio social: de un tiempo hasta ahora la imagen del profesorado se ha ido devaluando. Por un lado, algunas informaciones periodísticas han ofrecido un enfoque conflictivo de los educadores. Por otro, se han debilitado las relaciones entre padres y madres y el profesorado hasta el punto de que, a veces, parece que están enfrentados. Quizá se han depositado demasiadas expectativas en la escuela olvidando la responsabilidad y el impacto formativo de otras instituciones, y se culpa del "fracaso educativo" al profesorado (Vila, 2000).

- Las conductas antisociales de algunos alumnos y alumnas: Hay casos en los que la situación se vuelve insostenible y algunos profesores y profesoras que son objeto de desafíos, amenazas y agresiones temen ir al centro.

- El sistema de promoción y la remuneración no son del agrado de todos: A esto hay que añadir que algunos docentes trabajan con contratos precarios y carecen de la mínima estabilidad laboral, como es el caso de los funcionarios interinos que hemos nombrado anteriormente (Vila, 2000).
Los profesionales más propensos a padecer trastornos psíquicos son los que presentan inclinación a competir, alto nivel de aspiraciones, inseguridad, sentimientos de culpa y baja autoestima. Lo que es más preocupante, es que los profesores y profesoras que sufren estrés o depresión ocultan, en ocasiones, su problema.

La actividad educativa se realiza a través de la relación humana, que comporta a un tiempo enriquecimiento personal y compromiso emocional. Es innegable que el trato con el educando puede reportar muchas alegrías, pero también es cierto que la comunicación con el alumno o alumna conduce a veces a la frustración y la ansiedad. Hay profesores y profesoras que se ponen un escudo para protegerse y que se suele traducir en rigidez e inhibición, otros enferman ante los conflictos interpersonales y las múltiples responsabilidades.

Aunque no se llegue a la quiebra de la salud mental, lo que sí se observa en un significativo número de profesores y profesoras es una acumulación de malestar que lleva a vivir la actividad educativa como una mera vía de subsistencia, con lo cual ésta pierde su sentido y, en consecuencia, disminuye la calidad de la educación.

Dentro de las enfermedades fundamentalmente psicológicas, nos vamos a centrar más profundamente en el estrés, la ansiedad y la depresión que, en la mayoría de los casos, son las enfermedades más comunes entre el profesorado.

\section{El estrés:}

Tal y como el año pasado denunció el sindicato de enseñanza ANPE (Asociación Nacional del Profesorado Estatal), al menos ocho de cada diez profesores y profesoras presentan problemas anímicos. Estos números son -cuanto menos- sorprendentes, sobre todo porque muchos/as de ellos/as no lo afrontan ni reconocen públicamente, con lo cual se agrava más este problema (Bastante, 2008).

Esta situación es preocupante ya que cada vez son más los casos de absentismo, intención de abandonar la profesión, invalidez permanente o jubilación anticipada en los docentes (Vila, 2000). Si partimos de la base de que el papel de éstos, junto con la labor de los progenitores, es vital para la educación de las nuevas generaciones y que los roles del profesorado en la actualidad están siendo cambiados, perdiendo su posición de autoridad y reconocimiento, nos 
daremos cuenta de la gravedad del problema, pues nos encontramos con un colectivo docente que encuentra obstáculos a la hora de desempeñar su trabajo siendo preocupantes los problemas de salud psíquica. Estas dolencias se consideran actualmente como propias de la actividad docente (López López-Menchero, 2009).

Son diversas las causas que producen en el profesorado estrés, es decir, "sensación de que su situación laborar supone una amenaza para su autoestima y bienestar psíquico” (G.S.S.L.D., 2009). Algunas de las causas son:

- Sobrecarga de tareas: El docente trabaja no sólo intelectualmente, sino que debe programar sus clases realizando un ajuste de la materia y el tiempo del que dispone, da clases sobre diferentes temas, tiene en cuenta los diferentes intereses y capacidades del alumnado, realiza trabajo administrativo y se lleva a casa cantidad de trabajo. Del mismo modo, le son asignadas tareas que podrían ser llevadas a cabo por otros profesionales especialistas y orientadores, pues dentro de una misma clase se encuentra con una amplia gama de alumnos y alumnas que poseen capacidades diferentes, lo que requerirá de una mayor planificación (Colegio de profesores de Chile, 2000).

- Ratio superior a 25/30 alumnos/as: La preocupación de los docentes por la calidad de la educación debido a encontrarse con una ratio superior a 25/30 alumnos también desencadena en estrés (Vila \& López Fernández, 2000).

- Alumnos y alumnas con bajo nivel de motivación: El profesorado suele sentirse frustrado al intentar convencer al alumnado para que trabaje sin conseguir resultados positivos, provocando en el docente un gran cansancio físico y emocional.

- Clima de convivencia en los centros: Actualmente se esta produciendo un gran deterioro en las relaciones profesor/a - alumno/a, profesor/a - profesor/a y profesor/a padres/madres (López Fernández, 2000).

- Profeso/a - alumno/a: la actitud del alumnado y el modo de comportarse con el profesor/a constituye una causa de estrés docente.

- Profesor/a - profesor/a: Cada profesor/a puede aportar un punto de vista distinto sobre la forma de hacer las cosas, lo que genera desacuerdos que llegan a deteriorar la relación entre los profesionales.

- Profesor/a - padres/madres: algunos padres y madres culpan al profesorado de los fracasos de sus hijos/as menores y cuando éstos/as adquieren buenos resultados no valoran que son fruto de su labor.

- Ausencia de reconocimiento social: En nuestros días, son muchos los cambios que está sufriendo el sistema educativo, lo que conlleva que el profesorado deba adaptarse, usar nuevas tecnologías, cambiar criterios y metodología, etc. Lo que debería suponer un reconocimiento de los profesionales de la educación en la realidad se traduce en una devaluación de su función. Algunos padres y madres atribuyen el fracaso de sus hijos/as al docente, mientras que los logros alcanzados por sus hijos/as en el proceso de aprendizaje solamente a sus hijos/as considerando que son muy inteligentes sin valorar en ningún momento la labor del docente, lo cual provoca la tendencia de perdida de ilusión y animo en este colectivo (Vila \& López Fernández, 2000).

- Nuevas tecnologías: La presencia de las nuevas tecnologías en los centros es hoy una realidad, lo que supone que el profesorado debe adaptarse a ellas a pesar de que muchas veces esta tarea resulta dificultosa, desarrollando incluso sentimientos de incompetencia ante el uso de dichos medios y de frustración ya que los alumnos y alumnas poseen un mayor dominio de los mismos.

\section{La ansiedad:}

Los trastornos de ansiedad tienen en común con el estrés la activación fisiológica a nivel autonómico y muscular (tasa cardiaca elevada, sudoración, sensación de mareo, tensión muscular muy alta, dolores de espalda y cuello, etc.).

Una característica destacable de la ansiedad es su naturaleza anticipatoria, es decir, la respuesta de ansiedad posee la capacidad de señalar un peligro o amenaza para el propio sujeto. La ansiedad es un componente presente en la mayor parte de los trastornos psicológicos y psicosomáticos y una de las principales causas por las que el profesorado acude al médico 
(López López-Menchero, 2009). Además de la alteración de las respuestas fisiológicas, aparecen alteradas algunas respuestas conductuales y también cognitivas, es decir, distorsiones en pensamientos, ideas, creencias, expectativas, etc. del sujeto que la padece.

Entre las alteraciones conductuales destacan las conductas de evitación o escape de la situación temida. En el caso de mantenerse el sujeto en la situación temida, aparecen alteraciones conductuales diversas como la inmovilidad, el temblor, la tartamudez, etc.

Por otra parte, a nivel cognitivo y subjetivo predominan los pensamientos e ideas irracionales de inutilidad, humillación, incapacidad de enfrentarse con la situación y superarla, los sentimientos de miedo, preocupación, nerviosismo y vergüenza. El componente cognitivo va a estar presente también en la depresión aunque de una forma más exagerada, frecuente e intensa.

Los trastornos de ansiedad se caracterizan por los siguientes patrones:

- La reacción del sujeto es desproporcionada ante las demandas de la situación.

- La reacción es involuntaria y no logra controlarla.

- El sujeto percibe que su miedo es irracional y su conducta no es adaptativa.

- El problema del sujeto está determinado por el contexto.

Estos trastornos de ansiedad pueden ser trastornos de pánico, fobia específica (tipo animal, tormenta, agua, alturas, sangre, etc.), fobia social, trastorno de estrés agudo, postraumático y de ansiedad generalizada, trastorno de ansiedad por condición médica general y trastorno inducido por sustancias y trastorno no especificado.

\section{La depresión:}

La depresión es el principal trastorno del estado de ánimo. Es el diagnóstico clínico que se emplea con más frecuencia y una de la condiciones de malestar psicológico más frecuente en seres humanos (López LópezMenchero, 2009).

Podemos decir que entre el personal docente, la depresión sería el trastorno más grave dentro de los problemas de salud mental que presenta el profesorado y también uno de los que provocan más bajas laborales en los centros docentes, de ahí su importancia.
Un sujeto depresivo puede presentar diferentes síntomas:

- Síntomas afectivos negativos: tristeza, infelicidad y abatimiento son los más habituales. A veces predomina la irritabilidad, sensación de vacío y nerviosismo.

- Síntomas motivacionales y conductuales: estado general de inhibición, apatía, indiferencia y disminución en la capacidad de disfrute.

- Síntomas cognitivos: la atención, la memoria y la concentración están afectadas.

En general, el sujeto deprimido hace una valoración negativa de sí mismo, del entorno y del futuro, y suele presentar síntomas físicos como el insomnio (del $70 \%$ al $80 \%$ de los pacientes depresivos lo padecen) y molestias corporales difusas (dolores de cabeza, náuseas, estreñimiento, visión borrosa, etc.). Otros docentes presentan síntomas interpersonales, que da lugar a un mínimo o nulo contacto social con los demás y pérdida de interés por la gente (Guerrero, 1996).

Los trastornos depresivos se presentan con mayor frecuencia en mujeres que en hombres. Los diferentes estudios interculturales han puesto de manifiesto que esta diferencia de sexo puede ser debido a factores genéticos o también puede reflejar la existencia de pautas y actitudes educativas diferentes entre niños y niñas, en su sentido más amplio.

El mayor riesgo de padecer un trastorno depresivo mayor, se presenta en mujeres jóvenes y adultas de 25 a 44 años. A partir de esta edad se invierte la relación y es más frecuente en el hombre. La edad media de comienzo es a los 35 años.

\section{Salud física.}

Por regla general, a los problemas de salud física que suele presentar el profesorado se les da menor importancia que a los problemas de salud mental, probablemente porque estos últimos son más graves y más persistentes. De cualquier forma, no hay que olvidar que el personal docente desarrolla con frecuencia enfermedades derivadas de las condiciones de trabajo. Vamos a centrarnos en las siguientes, por ser las más frecuentes:

\section{Trastornos de la voz:}

Un trastorno que afecta de lleno a los docentes es la afonía y la disfonía. La primera consiste en la pérdida total de la voz y la segunda 
en la pérdida parcial de la voz debido a una alteración en su calidad (G.S.S.L.D., 2009)

Las principales causas que provocan la afonía son:

- Enfermedades del aparato respiratorio: larigintis, faringitis, amigdalitis, tos persistente, alergias, nódulos, tumores, etc.

- Malos usos de la voz: forzarla demasiado por motivos de trabajo.

- Ingestión de productos tóxicos o irritantes: hábito de fumar, el alcohol, productos irritantes como la pimienta, el vinagre, etc. (Colegio de profesores de Chile, 2000).

- Causas nerviosas: Voz afónica, ronca, aguda, etc.

- Frío excesivo: el clima o el beber agua fría o helados pueden producir un cambio en la voz.

Las principales causas de la disfonía son:

- Laringitis aguda. Es la causa más frecuente de disfonía y ocurre por una inflamación de las cuerdas vocales debido a una infección viral o a un uso excesivo de la voz.

- Nódulos de cuerdas vocales. Aparecen en personas con un mal uso vocal, que hablan muy alto, durante demasiado tiempo, o con una mala técnica. Son muy frecuentes en profesores y profesoras.

- Pólipos de cuerdas vocales. Las causas son las mismas que para los nódulos, pero aquí el componente inflamatorio es mayor.

- Reflujo gastroesofágico. El reflujo de material gástrico, sobre todo durante la noche, puede producir irritación de las cuerdas vocales y disfonía. Ocurre con mayor frecuencia en personas mayores. La disfonía es matutina y va cediendo a lo largo de la jornada.

- Cáncer de laringe. Esta causa de disfonía justifica por si sola la identificación de otras causas de alteración de la voz aunque sean aparentemente banales. Hay que sospecharla sobre todo ante un paciente fumador. El tabaco es la principal causa de cáncer de laringe.

- Parálisis de cuerdas vocales. Por afectación del nervio recurrente debido a cirugía del tiroides o compresión consecuencia de tumoraciones, o sin causa aparente.

- Alergias o traumas de la laringe pueden ser otras causas que provocan este trastorno.
Además de los problemas de afonía y disfonía, muchos docentes presentan dolores de garganta debidos a diferentes motivos como pueden ser laringitis, faringitis, amigdalitis, tos persistente, alergias, nódulos, tumores, etc., por lo que se aconseja que no fuercen la voz y que tomen medidas preventivas y no sólo curativas.

\section{Problemas de la vista:}

Con el uso creciente profesional y privado de los ordenadores han aumentado también las molestias de la vistas de los usuarios. Ojos dolientes, secos e irritados, dolores de cabeza y una vista borrosa, son algunos de los síntomas del llamado "síndrome de la visión del ordenador", que se ha convertido en una de las molestias más frecuentes de los empleados/as de oficinas en los últimos años, pero también los docentes presentan estos síntomas debido a la gran cantidad de horas que dedican a la búsqueda de materiales y preparación de clases enfrente del ordenador.

Creemos que una medida preventiva de riesgos laborales que nos afecta es aquella que contempla el trabajo con equipos que incluyen pantallas de visualización (ordenadores), y que está recogida en el Real Decreto 488/1997, de 14 de abril (BOE nº 97, de 23 de marzo de 1997). La adopción de las medidas que se toman en dicho decreto evitaría en gran proporción los riesgos para la seguridad y la salud de los trabajadores y trabajadoras, o si ello no fuera posible, tales riesgos tratarían de reducirse al máximo.

$\mathrm{El}$ origen de estos problemas se encuentra en un abuso o un mal uso del aparato, y su solución pasa por seguir unas simples recomendaciones al alcance de cualquiera.

- Alternar el trabajo en el ordenador con otros tipos de actividades.

- Si es necesario, aumentar el tamaño de la letra.

- No trabajar más de una hora continuada frente al monitor. Si esto no es posible, hacer frecuentes pausas, cada una de 5 minutos. Durante estas breves pausas orientar la mirada a un objeto distante durante 20 segundos.

- Si se sienten molestias, apagar el ordenador y hacer que sus ojos descansen. Es recomendable cerrar y abrir los ojos en el aire fresco para aliviarlos.

- Los caracteres tienen que estar bien definidos, con un buen nivel de contraste con respecto al fondo, de tamaño suficiente $\mathrm{y}$ 
con un espacio adecuado entre los renglones.

- Trabajar con texto negro sobre fondo blanco. Procurar no abusar de los colores.

La ubicación del ordenador también es importante (G.S.S.L.D., 2009):

- Situar el monitor a una distancia de unos 50 a 60 centímetros.

- Colocar la parte superior de la pantalla a una altura similar a la de los ojos, o ligeramente más baja. El monitor queda, de ese modo, en la zona óptima de visión, comprendida entre los cinco y los 35 grados por debajo de la horizontal visual, desde la cual contemplará todo sin ningún esfuerzo, su vista no se resentirá y evitará posturas lesivas.

- Inclinar el monitor hacia abajo a unos 20 grados en ángulo respecto a su mirada recta.

- Utilizar un atril para los documentos. Colocarlo a una distancia equivalente a la pantalla y a su misma altura. De esta forma no bajará y subirá constantemente la cabeza para mirar, y reducirá la fatiga visual.

Por último, hay que tener en cuenta la luz con la que se trabaja. Es recomendable:

- Colocar la pantalla perpendicular a la ventana, nunca enfrente pues al levantar la vista se pueden producir deslumbramientos, ni de espaldas a ella, ya que los reflejos de la luz natural sobre el cristal son inevitables.

- Tratar de que el monitor o la lámpara de mesa no sean la única iluminación. Utilizar luz ambiental. Evitará que cada vez que desplace los ojos fuera de la zona iluminada tengan que adaptarse a unas condiciones muy diferentes de luz.

- No incurrir en el error frecuente de utilizar mucha luz. Muchas fuentes de luz en un sitio pueden desviar la atención de sus ojos del monitor o causar reflexiones en la pantalla.

- El exceso de luz también cansa la vista, es preferible orientar las lámparas para que iluminen el área de trabajo y no incidan directamente sobre los ojos o sobre la pantalla.

- Trabajar con monitores que incorporen un tratamiento antirreflejo o un filtro especial. Tener especial cuidado en que el filtro no oscurezca demasiado el monitor.
- La imagen de la pantalla ha de ser estable, sin destellos, reflejos, centelleos o reverberaciones. Según un estudio, el nivel de luminancia de los monitores de rayos catódicos es inestable durante los primeros 20 minutos tras el encendido, por lo que es aconsejable encender el monitor antes de empezar a trabajar con él: reducirá la fatiga visual producida por estas variaciones.

\section{Resfriados y gripes:}

Los resfriados y gripes comunes afectan en gran medida a los trabajadores y trabajadoras. Esto puede deberse a los cambios de temperatura que se producen fuera y dentro del centro, a una utilización excesiva de aire acondicionado o, por el contrario, de calefacción en invierno. El contraste de temperaturas al salir a la calle puede ser un agravante de que se produzcan estas enfermedades comunes (Vila, 2000).

Un resfriado con frecuencia comienza sintiendo cansancio, estornudando, tosiendo y con mucosidad en la nariz. Puede que no se tenga fiebre o puede que se tenga fiebre baja: justo uno o dos grados más de lo normal. Probablemente también se tengan dolores musculares, ronquera o dolor de garganta, ojos llorosos y dolor de cabeza.

La gripe comienza de repente y de forma más dura. Normalmente las personasque la padecen se sienten débiles y cansadas, con fiebre, tos seca, nariz con mucosidad, escalofríos, dolores musculares, dolor de cabeza intenso, dolor en los ojos y dolor de garganta. Usualmente se toma más tiempo para mejorarse de la gripe que de un resfriado.

Las recomendaciones principales para curar los resfriados y las gripes son:

- Quedarse en casa y descansar, especialmente mientras se tiene fiebre.

- Dejar de fumar y evitar el humo de las personas que fuman a su alrededor, el cual puede empeorar los síntomas de su resfriado.

- Tomar líquidos en abundancia como agua, jugos de frutas y sopas claras. Los líquidos ayudan a aflojar la mucosidad. Los líquidos también son importantes si se tiene fiebre pues la fiebre puede hacer que sus líquidos se evaporen lo cual puede ocasionar una deshidratación.

- No beber alcohol.

- Hacer gárgaras con agua con sal varias veces al día para aliviar el dolor de garganta. 
Los aerosoles para la garganta y las pastillas para la garganta también pueden ayudar a aliviar el dolor.

- Usar gotas para la nariz de solución salina o agua con sal para ayudar a aflojar el moco y humedecer al área sensible dentro de su nariz.

\section{Gastroenteritis y problemas estomacales:}

Las gastroenteritis pueden deberse a múltiples motivos. La más común es un virus que se propaga rápidamente en diferentes ambientes a través del agua, alimentos contaminados o de persona a persona. La ingestión de hongos o de frutas silvestres no comestibles que contienen sustancias tóxicas, así como los excesos en la comida $\mathrm{o}$ en la bebida, $\mathrm{y}$ de ciertos medicamentos, como las aspirinas, laxantes, etc. puede dar lugar a distintos tipos de gastroenteritis. Las personas que sufren alguna alergia y no lo saben pueden sufrir intolerancia frente a algunos alimentos y esto producirles gastroenteritis.

Otros problemas estomacales pueden ser los cortes de digestión, la inapetencia alimenticia, la acidez estomacal, los gases, etc. Estos últimos son más frecuentes e incómodos, y pueden ser debidos a dietas caóticas, estreñimiento, comer demasiado rápido, dietas con exceso de fibra, e incluso nerviosismo.

\section{Sobrecarga muscular y problemas de espalda:}

El trabajo permanente en posturas o posiciones incómodas y permanecer excesivo tiempo de pie puede provocar sobrecarga muscular y otros problemas tales como el dolor de espalda, la tensión en el cuello, el dolor de cabeza generado por la tensión muscular, hinchazón, dolor de piernas y tobillos, varices, etc. (G.S.S.L.D., 2009). Por lo que es aconsejable ser conscientes en todo momento de tomar hábitos como cambiar de postura y de tarea tanto cuando estamos de pie como sentados.

\section{Dolor de cabeza:}

El dolor de cabeza es una molestia del cuero cabelludo o el cuello y sus causas con muy diversas. La mayoría de las personas con dolores de cabeza se pueden sentir mucho mejor haciendo cambios en su estilo de vida, aprendiendo formas de relajarse y ocasionalmente tomando medicamentos.

Los dolores de cabeza más comunes probablemente son causados por contracción y tensión muscular en los hombros, el cuello, el cuero cabelludo y la mandíbula. Estos se denominan cefaleas tensionales. Estos dolores de cabeza a menudo están relacionados con el estrés, la depresión o la ansiedad, trastornos, como hemos dicho, que afectan al profesorado en gran medida. El hecho de trabajar demasiado, no dormir lo suficiente, omitir comidas y consumir alcohol o drogas psicoactivas puede hacer que seamos más susceptibles a dichos dolores. Igualmente, los dolores de cabeza pueden desencadenarse por el chocolate o el queso. Las personas que consumen cafeína pueden presentar dolores de cabeza cuando no obtienen la cantidad diaria usual.

\section{Problemas alérgicos:}

$\mathrm{La}$ alergia es una hipersensibilidad a una particular sustancia que, si se inhala, ingiere o se toca produce unos síntomas característicos. Las alergias más comunes son las siguientes: alergia al polen, los ácaros del polvo, los animales domésticos, los hongos, algunos alimentos, picaduras de insectos, medicamentos, etc.

Aunque las alergias se den de manera frecuente en muchas personas, hay que decir que sus síntomas son desagradables y, en ocasiones, pueden provocar un peor o nulo desempeño de las labores de los profesionales, en este caso, del personal docente.

Algunos de estos síntomas son lagrimeo y picor de ojos, secreción nasal, estornudos, congestión nasal, picor y enrojecimiento de la piel, tos, ahogos, dificultad respiratoria, mareos, etc.

\section{Factores de riesgo en las aulas.}

Aunque en un menor grado, también es importante que los docentes tengan presente que existen factores que pueden perjudicar su salud cuando están trabajando en las instalaciones del centro y en las aulas (Colegio de profesores de Chile, 2000 \& Pérez Soriano, 2005).

Estos factores pueden ser una mala iluminación del lugar de trabajo, una temperatura y humedad inadecuadas, la existencia de ruidos exteriores o provenientes del mismo centro (G.S.S.L.D., 2009), mobiliario e infraestructuras inadecuados tanto para profesores y profesoras como para alumnos y alumnas, instalaciones eléctricas que dan problemas, servicios higiénicos no satisfactorios, e incluso accesos al centro que no cubran las necesidades de todo el personal del centro. 


\section{4.- POSIBLES SOLUCIONES PARA LA SALUD LABORAL DOCENTE.}

La mejor estrategia en la mejora de la salud laboral de los docentes es a través de la Prevención de Riesgos Laborales. La prevención significa anticiparse y actuar antes de que algo suceda con el fin de impedirlo o para evitar sus efectos (López López-Menchero, 2009).

De forma general poder decir que algunas de las soluciones para los problemas de salud laboral docente que se han planteado anteriormente podrían ser: mantener el orden y la limpieza en el entorno de trabajo, adoptar técnicas para la reducción del estrés, concienciarnos y sensibilizarnos con campañas anti-tabaco, mantener un entorno social satisfactorio, seguir formando al profesorado y al personal laboral y funcionario del centro, trabajar con un mobiliario adaptado a nuestras necesidades con mesas y sillas ergonómicas, utilizar técnicas para la correcta utilización de la voz, etc.

La satisfacción laboral es necesaria para que cualquier trabajador/a se entusiasme con la tarea y rinda. En el caso de los educadores y educadoras, el bienestar, además de fuente de salud, se proyecta sobre el alumnado. Los profesores y profesoras que están a gusto con su labor infunden en los alumnos y alumnas un estado de ánimo favorable para la actividad que se realiza.

Algunas pautas preventivas del malestar docente pueden ser (Martínez-Otero, 2003):

- Disponer de un proyecto educativo que provoque el crecimiento como personas y profesionales, y dar entrada en el mismo a la participación de los demás. El espíritu de comunidad refuerza la energía vital y protege la personalidad.

- Aprender a disfrutar de pequeños logros, así como a advertir nuevos y buenos matices en la cotidianidad.

- Programar y aprovechar los períodos vacacionales, para salir del circuito de tensión y reincorporarse al trabajo con fuerzas renovadas. Adquiere gran importancia la utilización saludable del tiempo libre.

- Entre las medidas protectoras del malestar no pueden faltar tampoco la actitud mental positiva, la dieta equilibrada, la actividad física y la relajación muscular.
- Intercambiar experiencias y opiniones con los y las colegas. La comunicación y las relaciones personales presididas por la cordialidad neutralizan los aspectos negativos del trabajo. El aislamiento a que a veces propende el profesor o profesora no hace sino acrecentar los problemas.

- Poner una nota de creatividad en lo que se realiza. La actividad educativa es susceptible de innovación. Es verdaderamente lamentable el caso de algunos docentes que, apoyándose en unos apuntes amarillos y desgastados, se limitan a repetir curso tras curso los mismos contenidos. Esto no sólo desmotiva al alumnado sino al propio docente.

Hay que mencionar el relevante papel que juega el centro educativo en su conjunto. El clima institucional de confianza, seguridad, respeto y valoración es una de las mejores vacunas contra el malestar, así como uno de los principales impulsores del crecimiento individual y colectivo. El apoyo de la organización, tanto en el plano del reconocimiento profesional como en el de las retribuciones, constituye un objetivo para garantizar la adaptación laboral y el equilibrio personal de los docentes.

A continuación analizaremos las posibles soluciones que debería adoptar el personal docente y no docente de los centros educativos para prevenir y solventar los problemas de salud física y mental que hemos comentado en los apartados anteriores.

En el caso de la sobrecarga de tareas que tiene en ocasiones el profesorado y que le produce serios problemas de ansiedad y estrés, sería preciso establecer períodos de descanso y alternancia en las tareas (Colegio de profesores de Chile, 2000). En este apartado también habría que añadir que el trabajo del profesorado debe ser bien organizado y que la ratio de más de 25/30 alumnos en su clase no se debe dar. Para ello, habría que adecuar el número de alumnos y alumnas al grupo-clase no superando la ratio (Vila \& López Fernández, 2000).

Para conseguir una mayor motivación en el alumnado, se debería proporcionar al profesorado una formación psicológica que le lleve a su autoconocimiento personal y a la adquisición de habilidades que le permitan motivar al grupoclase. Como hemos dicho anteriormente, es fundamental que se cree en la escuela un clima 
agradable de convivencia. Para ello, sería conveniente formar al profesorado a través de programas con el fin de adquirir conocimientos que faciliten su convivencia en los centros con los agentes que intervienen en el proceso educativo. La creación de una escuela de padres y madres en la que se transmitan valores que resalten la labor docente sería una solución muy interesante. En el caso del alumnado, este podría desarrollar programas para la mejora de la convivencia (López Fernández, 2000).

En cuanto al reconocimiento social del profesorado, una buena idea es que se intervenga desde asociaciones, sindicatos, organizaciones, etc. con el fin de que la sociedad tome conciencia de la importancia de la función docente (Vila \& López Fernández, 2000).

La cuestión de las Nuevas Tecnologías quedaría resuelta si se desarrollaran programas de formación del profesorado en el ámbito de las Nuevas Tecnologías con el fin de que adquieran un adecuado dominio de las mismas.

Algunas de las estrategias que hemos planteado para afrontar el problema del estrés podrían ser que los docentes (Bastante, 2008 \& López López-Menchero, 2009):

- Realicen ejercicio físico tras la jornada escolar, pues acabarán mentalmente agotados pero físicamente activos, sus cuerpos estarán en tensión y necesitarán ejercicio físico para liberar esa tensión y relajarse.

- Adquieran el aprendizaje de técnicas de relajación y autocontrol.

- Aprendan técnicas de resolución de conflictos.

- Desarrollen habilidades sociales, motivacionales, asertivas y de comunicación, así como dinámicas de grupos.

- Realicen una adecuada organización del tiempo de trabajo.

- Planifiquen actividades de ocio y tiempo libre.

- Se apoyen entre sí, con amistades y familiares.

- Desarrollen programas y actividades destinadas al tratamiento y la prevención del estrés.

Frente al tema de la sobrecarga muscular en el personal docente y no docente, podríamos tomar las siguientes medidas cuando estemos de pie (G.S.S.L.D., 2009):
- Alternar esta postura con otras que impliquen movimiento. Por ejemplo, si nos encontramos dando una explicación, no quedarnos de pie en un solo punto del aula, sino caminar entre los pupitres e ir de un lado a otro, con pasos lentos para no perturbar la atención de los estudiantes y la modulación de su voz, pero asegurándonos de movernos. Esto nos ayudará también a mejorar la atención de nuestra clase, pues los estudiantes se sentirán parte de la explicación al acercarnos físicamente a ellos.

- Repartir el peso del cuerpo en ambas piernas. Muchas veces sentimos alivio cuando cargamos nuestro peso en una pierna más que en la otra. Esta postura sólo logrará tensionar el músculo del que se está abusando y causará más cansancio posteriormente. Cuando estemos de pie, se debe tratar de hacerlo con la espalda recta, la frente en alto y las piernas separadas, sosteniendo la misma cantidad de peso con ambas.

Cuando nos encontremos sentados deberemos:

- Apoyar siempre la espalda al respaldar de la silla, asegurándose de que ésta se encuentre recta, sin arquear y con los hombros echados hacia atrás.

- Mientras estemos sentados, deberemos rotar los tobillos, hacia adentro y hacia afuera, estirar las piernas, y subir y bajar la punta de los pies.

- Apoyar ambos pies en el suelo y procurar nunca cruzar las piernas.

El problema de los dolores de cabeza son trastornos muy molestos y que impiden al personal docente y no docente realizar su labor. Por ello es importante seguir hábitos saludables que puedan disminuir el estrés y reducir la probabilidad de padecer dolores de cabeza. Estos son:

- Dormir bien.

- Llevar a cabo una dieta saludable.

- Hacer ejercicio de manera regular.

- Estirar el cuello y la parte superior del cuerpo, especialmente si el trabajo implica utilizar el ordenador.

- Aprender a adoptar una postura apropiada.

- Dejar de fumar. 
- Aprender a relajarse utilizando la meditación, la respiración profunda, el yoga u otras técnicas.

- Usar gafas apropiadas, de ser necesario.

Por último, trataremos el tema de las alergias que se dan con mucha frecuencia entre los trabajadores y trabajadoras. Para mejorar nuestra salud en este respecto, el paciente debe fijarse en las condiciones ambientales que le rodean (residencia, trabajo, contacto con animales, etc.), y en los factores desencadenantes de los síntomas, calendario de síntomas, aficiones $\mathrm{y}$ antecedentes familiares de alergia. De esta manera podrá identificar el tipo de alergia que padece. En general deben evitarse aquellas situaciones que de forma reiterada nos provoquen reacciones alérgicas.

En cuanto a los trastornos de la voz que afectan de lleno al personal docente (G.S.S.L.D., 2009), generalmente debido a su trabajo en las aulas, se deberían tener en cuenta las siguientes consideraciones para reducir el desgaste fonatorio en el docente:

- Utilizar una correcta técnica fonatoria teniendo en cuenta la respiración, la relajación y la resonancia.

- Evitar cualquier esfuerzo exagerado en el mecanismo de emisión de la voz.

- Hablar menos, preguntar más y dejar explicar a los demás.

- No gritar en la clase.

- Articular claramente sin exageraciones.

- Permitir que los alumnos y alumnas den clases especiales o hagan presentaciones de temas específicos para hacer menor uso de la voz.

- No abrigarse en exceso para no transpirar y generar disfonía.

- Respetar las puntuaciones.

- Mantener una postura correcta en clase.

- Relajar las cuerdas vocales tomando té tibio con limón y azúcar.

- Evitar la excesiva calefacción o aire acondicionado ya que seca la mucosa bucal.

- Utilizar ropa cómoda sin ajustarse la zona abdominal.

\section{5.- CASO CONCRETO DE NUESTRA ESCUELA.}

Después de haber estudiado el estadillo mensual de ausencias por motivos de salud en nuestro puesto de trabajo, hemos comprobado que, aunque se han producido pocas ausencias debidas a esta causa, asombrosamente, el 50\% de esas ausencias por enfermedad han sido provocadas por problemas de gastroenteritis entre el personal docente y no docente de nuestra escuela, y han tenido una duración máxima de tres días en cada caso; el resto de los casos de enfermedades de corta duración han sido por problemas alérgicos y de cirugía menor.

Por el contrario, hemos contado con dos casos de enfermedades de larga duración en la escuela, que no han persistido durante más de doce días, y ha sido el caso de una operación y gripe común.

Resulta interesante que todas las ausencias han sido resultado de problemas de salud física y en ningún caso hemos tenido problemas de salud mental importantes aunque no podemos negar que, en ocasiones, el personal docente ha vivido situaciones de estrés debidas, sobre todo, a la elaboración de documentos del centro dentro de plazos establecidos y otras tareas administrativas, pero en ningún caso nos hemos visto sobrepasados por el trabajo docente en sí. En cualquier caso, estas situaciones siempre han sido muy puntuales y nunca han provocado una falta de asistencia del personal al centro de trabajo.

Hemos comprobado que entre los problemas de salud física más frecuentes en nuestro centro se han dado problemas estomacales, alergias, trastornos de la voz (disfonía), gripes y dolores de cabeza. Pero sólo de forma ocasional en el caso de los problemas estomacales y las gripes, el personal ha faltado a su puesto de trabajo. En este punto tenemos que tratar el tema del acondicionamiento en el centro. En los meses de invierno hemos contado con calefacción en el centro, pero durante los meses más calurosos hemos notado de una forma considerable que el profesorado no rinde de la misma manera que antes en su trabajo debido a la falta de aire acondicionado en la escuela. Aunque pueda parecer un hecho sin importancia, esto ha provocado que los profesores y profesoras pospongan tareas de la escuela que debían realizar en su horario para hacerlas en casa para poder contar con un acondicionamiento mejor, ya que el sofoco en el centro de trabajo les ha producido dolores de cabeza y agobios que no han permitido que pudieran desarrollar el trabajo de una forma rápida y eficaz.

Es importante mencionar el mayor problema que hemos tenido en el centro $y$ que ha 
provocado las mayores tensiones y momentos de estrés entre el personal docente: la falta de un lugar de trabajo adaptado a nuestras necesidades. Nuestro personal no docente no ha tenido grandes problemas, pero sí el personal docente. Hemos contado con una sala pequeña con mobiliario para la Directora y la Jefa de Estudios, es decir, dos mesas con ordenadores que hemos tenido que compartir con el resto del profesorado. Esta situación ha causado muchos problemas de estrés y descoordinación entre los profesores y profesoras (López López-Menchero, 2009), a la vez que un bajo rendimiento en su trabajo. Conforme el centro vaya contando con nuevo profesorado este problema se irá incrementando y la situación se hará todavía más insostenible, por lo que la búsqueda de soluciones frente a este tema se hace urgente.

En cuanto al alumnado, por regla general, los profesores y profesoras no han tenido ningún problema que afectara a su salud laboral docente, ya que, por suerte, nuestros alumnos y alumnas han sido, en su mayoría, personas adultas, con mucha motivación, con ganas de aprender, muy participativas e involucradas $\mathrm{y}$, sobre todo, personas que han valorado el hecho de tener una Escuela Oficial de Idiomas a su alcance y no han querido desaprovechar esta oportunidad.

Por otro lado, debemos mencionar el caso de algunos grupos con alumnado más exigente (probablemente porque muchos de ellos son profesores o profesoras en otros centros o personas con un nivel educativo elevado) que ha provocado que el profesorado se esforzara todavía más, si cabe, para no defraudar a su alumnado. Sólo de forma puntual, tenemos que nombrar a ciertos alumnos y alumnas adolescentes que han causado un retardo en el ritmo de las clases y han mostrado un menor grado de motivación que, en ocasiones, ha causado que el profesorado se replanteara sus clases para que su labor se produjera de una forma amena y satisfactoria.

Después de haber entrevistado a todo el profesorado del centro acerca de estos temas, hemos coincidido en que sería conveniente llevar a la práctica una propuesta que hemos realizado por primera vez en nuestra escuela. Creemos que para que los profesores y profesoras estén actualizados/as y tengan feedback de sus alumnos y alumnas es fundamental que éstos/as realicen un cuestionario anónimo por trimestre. De esta forma, y con cuestiones muy simples en castellano, los alumnos y alumnas harán saber a su profesor/a lo que opinan sobre ciertos temas que le incumben. Así, éste/a podrá hacer cambios en lo que crea conveniente y mejorar de forma profesional lo que es fundamental para una buena salud mental.

Al final de este artículo se adjunta el cuestionario real (anexo 1) que nuestros/as alumnos/as realizaron tras la finalización del primer trimestre del curso académico. Conforme van pasando los trimestres, las cuestiones varían un poco, preguntándoles ya al final de curso en tiempo en tiempo pasado y no en presente. Por ejemplo: “¿Cómo ha ido el curso?”, “¿Has alcanzado tus objetivos al final del curso?”, etc. Esta es la mejor manera para que el alumnado reflexione sobre su trabajo en el curso, lo que ha conseguido y lo que le ha costado más, pero también es interesante para que el profesorado mejore algunas cuestiones en próximos cursos y que se evalúe como docente. De esta manera, siempre se dará en la escuela un ambiente fresco y renovado frente a la labor de enseñanzaaprendizaje.

\section{6.- ENCUESTA REALIZADA PERSONAL DEL CENTRO.}

\section{Descripción de la encuesta}

Creemos que el mejor punto de partida a la hora de realizar este artículo era investigar el trabajo en la escuela y los problemas de salud física y mental que afectaba al personal docente y no docente para poder detectar las necesidades más urgentes que se presentaban en el centro. Para ello, hemos elaborado una encuesta anónima sobre los puntos más importantes que se desarrollan en el artículo.

La primera parte de la encuesta (anexo 2) se centra en el trabajo que el personal desempeña en el centro y de las condiciones laborales de cada uno de nosotros/as. El personal sólo tendrá que marcar del 0 al 5 la puntuación que más se ajuste a su satisfacción con los asuntos concretos, siendo el 0 la menor puntuación y el 5 la mayor puntuación.

La segunda parte de la encuesta trata los problemas de salud mental y física que afectan al personal de nuestra E.O.I. Algunos de esos problemas de salud son conocidos por el resto del personal, pero otros han sido más sorprendentes. Al ser la encuesta anónima, el personal se ha sentido libre para responder con franqueza, lo cual era el objetivo primordial de la encuesta, 
porque así podremos buscar soluciones apropiadas y eficaces a esos problemas. Dentro de esta misma encuesta, el personal docente y no docente propondrá también las soluciones más acertadas que, desde su punto de vista, serían aconsejables considerar en nuestra escuela.

\section{Resultados}

Una vez finalizada la realización la encuesta sobre la importancia de la Salud Laboral Docente entre el personal del centro, vamos a comentar y analizar los resultados obtenidos.

Mientras que el personal no docente está medianamente conforme con que su lugar de trabajo es adecuado para desarrollar bien su labor, el $100 \%$ de los docentes ha coincidido en que el lugar de trabajo fuera de las aulas no es adecuado, y dentro de las aulas podría serlo más.

La totalidad del personal de la E.O.I. ha afirmado que está de acuerdo con las condiciones de trabajo este año (horarios, grupos en el caso del profesorado, etc.) y que ha existido una relación de cordialidad entre todos los miembros.

El 80\% del personal ha admitido que hemos tenido un gran número de jornadas y cursos de formación a nuestra disposición. Habría que intentar que para el próximo año todo el personal estuviera satisfecho en cuanto a la formación ofrecida.

Son muy interesantes los resultados del personal docente y no docente del centro en cuanto a los conocimientos que tenemos sobre primeros auxilios y prevención de riesgos laborales, ya que el $100 \%$ de nosotros/as hemos dado la menor puntuación a estos asuntos, lo que quiere decir que hay una grave falta de información en cuanto a estos temas y que sería muy recomendable solventar esta situación.

El $80 \%$ del personal ha manifestado que le preocupan los problemas de salud mental y que sería interesante contar con más asesoramiento para su prevención. El $60 \%$, por su parte, ha manifestado que le preocupan los problemas de salud física, lo cual podría quedar solventado en gran medida contando con mejores infraestructuras y mobiliario en la escuela. Es importante añadir que el $90 \%$ del profesorado considera que ha sido muy conveniente prestar atención y reflexionar acerca de los problemas de salud que afectan al personal del centro.

Entre los problemas de salud física más frecuentes presentados entre el personal docente y no docente de nuestro centro se encuentran, en este orden, las sobrecargas musculares, problemas posturales, dolores de cabeza, trastornos en la voz y problemas alérgicos. Los problemas de salud física que afectan en menor medida a nuestro personal son los siguientes: problemas de tensión arterial, problemas de visión, dolores de garganta, gastroenteritis y resfriados.

A continuación parafrasearemos algunas de las soluciones que surgen desde el personal de nuestro centro para solventar estos problemas: "Sería conveniente que se produjera una mejora en las instalaciones del centro", "Necesitamos más material adaptado a las necesidades del centro y más consejos a la hora de adoptar las posturas adecuadas para evitar sobrecargas musculares y problemas de voz", "Sería interesante disponer de WIFI para trabajar en las aulas y no congregarnos todos los profesores/as en un pequeño despacho", "Sería recomendable conocer pautas de relajación", "Sería necesario aclimatar las estancias". Así, podemos concluir que lo más importante para el personal del centro es ampliar nuestros espacios de trabajo para desarrollar mejor nuestra labor y no perjudicar al resto de los profesores $y$ profesoras.

Entre los problemas de salud mental más frecuentes que presenta nuestro personal se encuentran, en este orden, la angustia debido a la inseguridad laboral (como es el caso de los/as interinos/as del centro), el estrés, la ansiedad, y el nerviosismo. Los problemas de salud mental que afectan en menor medida e incluso de forma nula a nuestro personal son los siguientes: la presión debido al exceso de responsabilidad, la depresión, la falta de autoestima, la frustración, el insomnio y la desestabilización emocional.

Algunos de los comentarios que han planteado el personal docente y no docente del centro para encontrar soluciones han sido: "Necesitamos formación en cuanto a la prevención del estrés", "Que exista siempre una comunicación abierta y transparente entre compañeros/as y que se siga buscando el equilibrio entre el buen ambiente de trabajo y los intereses personales", "Es fundamental que el Equipo Directivo cuente con más asesoramiento en cuanto a las tareas propias de este equipo para evitar situaciones de estrés y la ansiedad". Por ello, podemos concluir que para el curso próximo sería aconsejable recibir asesoramiento y formación por parte de profesionales para todos 
los tipos de necesidades que puedan surgir en el centro.

\section{7.- CONCLUSIONES.}

A modo de conclusión cabría decir que gracias a este artículo acerca de la importancia de la Salud Laboral Docente, el personal del centro ha aprendido a reflexionar sobre sus condiciones de trabajo y a mejorarlas considerablemente.

Creemos que el sistema educativo debe jugar un papel fundamental en la instauración de una cultura de prevención de los riesgos laborales en nuestra sociedad. Ello requiere que el impulso de esa cultura preventiva esté presente en todos los niveles educativos (López Fernández, 2000). Por lo que se deberá integrar la prevención y la promoción de la salud laboral, así como la reducción de la siniestralidad laboral y la mejora de las condiciones de trabajo del personal docente a su cargo, eliminando o reduciendo los riesgos de accidentes y enfermedades y, con ello, la disminución de lesiones a personas y de daños materiales que aquellos suelen ocasionar.

El personal de la E.O.I. cree que el mejor punto de partida para prevenir los problemas de salud en el centro es mantener siempre unas buenas condiciones y ambiente de trabajo, donde el diálogo, la cooperación y el respeto constituyan los tres pilares fundamentales de nuestro estilo de vida en la escuela.

Según la Organización Mundial de la Salud, "estilo de vida es una forma de vida que se basa en patrones de comportamiento identificables, determinados por la interacción entre las características personales individuales, las interacciones sociales y las condiciones de vida socioeconómicas y ambientales” (OMS, 1999).

\section{8.- REFERENCIAS BIBLIOGRÁFICAS.}

Acuerdo de 19 de septiembre de 2006, del Consejo de Gobierno, por el que se aprueba el I Plan Andaluz de Salud Laboral y Prevención de Riesgos Laborales del personal docente de los centros públicos dependientes de la Consejería de Educación (2006-2010). (BOJA n ${ }^{\circ} 196$ de 9 de octubre de 2006). Sevilla.

Bastante, J. (2008). Ocho de cada diez profesores sufren ansiedad [en línea]. Disponible en: http://www.publico.es/espana/174041/. Consulta: 2009, 18 de octubre].

Colegio de Profesores de Chile A.G. (2000). "Estudio de la Salud Laboral de los educadores de Chile" [en línea]. Disponible en: http://200.27.90.155/index.php?option=content\&t ask=view\&id=275. [Consulta: 2009, 2 de agosto].

Decreto 117/2000, de 11 de abril, por el que se crean los servicios de Prevención de Riesgos Laborales para el personal al servicio de la administración de la Junta de Andalucía. (BOJA n ${ }^{\circ}$ 45, de 15 de abril de 2000).

Gabinete de Seguridad y Salud Laboral Docente (2009). Riesgos profesionales docentes. Consejería de Educación - Junta de Andalucía [en línea]. Disponible en: http://www.juntadeandalucia.es/educacion/salud/ com/jsp/index.jsp. [Consulta: 2009, 26 de julio].

Guerrero Barona, E. (1996). "Estrés, ansiedad y depresión: Incidencia en el profesorado” [en línea]. Disponible en: http://www.aidex.es/estres/articuloseloisa/eloisa1 .htm. [Consulta: 2009, 18 de julio].

Ley 31/1995, de 8 de noviembre, de Prevención de Riesgos Laborales. Jefatura del Estado. (BOE $\mathrm{n}^{\circ}$ 269, de 10 de noviembre de 1995). Madrid.

López Fernández, J. (2000). "Estudio sobre la salud laboral del profesorado”. MUFACE Educación [en línea]. Disponible en: http://www.map.es/gobierno/muface/v179/educ.h tm. [Consulta 2009: 19 de septiembre].

López López-Menchero, J.L. (2009). La salud Laboral de los docentes [en línea]. Disponible en:

http://www.educaweb.com/noticia/2009/03/26/sa lud-laboral-docentes-13542.html. [Consulta: 2009, 7 de octubre].

Martínez-Otero, V. (2003). La salud mental del profesorado. Tribuna Comunidad Escolar 713 [en línea]. Disponible en: http://comunidadescolar.cnice.mec.es/713/tribuna.html. [Consulta: 2009, 30 de septiembre].

Pérez Soriano, F.J. (2005). Prevención de Riesgos Laborales en centros docentes [en línea]. Disponible en: http://www.prevenciondocente.com/riesgo.htm. [Consulta: 2009, 24 de octubre].

Pérez Soriano, F.J. (16 de enero de 2007). Periódico El País. La seguridad se aprende en la escuela [en línea]. Disponible en: http://personal.telefonica.terra.es/web/sorpejav13 /elpais1601.pdf. [Consulta: 2009, 25 de agosto].

Real Decreto 488/1997, de 14 de abril, sobre disposiciones mínimas de seguridad y salud relativas al trabajo con equipos que incluyen pantallas de visualización. (BOE n 97, de 23 de marzo de 1997). Madrid.

Vila, D. (2000). Estudio sobre la salud laboral del profesorado. MUFACE Educación [en línea]. Disponible

en: http://www.map.es/gobierno/muface/v179/educ.h tm. [Consulta 2009: 19 de septiembre]. 


\section{ANEXOS:}

Anexo 1.- Encuesta fin del primer trimestre del curso.

Anexo 2.- Encuesta al personal del centro.

ANEXO 1: ENCUESTA FIN DEL $1^{\text {er }}$ TRIMESTRE DEL CURSO

\section{ENCUESTA}

1. ¿Cómo está yendo el curso? ¿Se están cumpliendo tus expectativas?

2. ¿Qué destrezas te causan mayor esfuerzo (comprensión oral, comprensión escrita, expresión oral o expresión escrita)? ¿Cuál te gusta más?

3. ¿Cuál crees que es la mejor manera de trabajar en clase (individualmente, en parejas o en grupos)? ¿Por qué?

4. ¿'Te sientes animado o frustrado? ¿Por qué?

5. ¿Te gusta la forma en la que la profesora está impartiendo las clases? ¿Por qué? ¿Mejorarías algo?

6. ¿Te gusta la forma en que la lectora está impartiendo las clases? ¿Por qué? ¿Mejorarías algo?

7. ¿Cómo es el ambiente en clase? ¿Te sientes libre y confiado para intervenir?

8. ¿Crees que serás capaz de alcanzar tus objetivos al final del curso? 
PERSONAL DOCENTE

PERSONAL NO DOCENTE

\begin{tabular}{|c|c|c|c|c|c|}
\hline & 1 & 2 & 3 & 4 & 5 \\
\hline $\begin{array}{l}\text { 1. Mi lugar de trabajo (fuera de las aulas) es ade- } \\
\text { cuado para desempeñar bien mi labor }\end{array}$ & & & & & \\
\hline $\begin{array}{l}\text { 2. Mi lugar de trabajo (en las aulas) es adecuado } \\
\text { para desempeñar bien mi labor }\end{array}$ & & & & & \\
\hline $\begin{array}{l}\text { 3. Estoy de acuerdo con la distribución de mis } \\
\text { grupos y mi horario este curso }\end{array}$ & & & & & \\
\hline $\begin{array}{l}\text { 4. Considero que existe una relación de concordia } \\
\text { entre todo el personal de la EOI (docente y no } \\
\text { docente) }\end{array}$ & & & & & \\
\hline $\begin{array}{l}\text { 5. He tenido un gran número de jornadas y cursos } \\
\text { de formación a mi disposición }\end{array}$ & & & & & \\
\hline $\begin{array}{l}\text { 6. Tengo conocimientos acerca de los primeros } \\
\text { auxilios }\end{array}$ & & & & & \\
\hline $\begin{array}{l}\text { 7. Tengo conocimientos acerca de la prevención } \\
\text { de riesgos laborales en mi puesto de trabajo }\end{array}$ & & & & & \\
\hline $\begin{array}{l}\text { 8. Me preocupan los problemas de salud física } \\
\text { (trastornos de la voz, de la vista, estar excesivo } \\
\text { tiempo de pie, etc.) }\end{array}$ & & & & & \\
\hline $\begin{array}{l}\text { 9. Me preocupan los problemas de salud mental } \\
\text { (estrés, depresión, falta de autoestima, insom- } \\
\text { nio, etc.) }\end{array}$ & & & & & \\
\hline $\begin{array}{l}\text { 10.Una buena solución para los problemas de sa- } \\
\text { lud física sería contar con mejores infraestruc- } \\
\text { turas y mobiliario }\end{array}$ & & & & & \\
\hline $\begin{array}{l}\text { 11. Una buena solución para los problemas de sa- } \\
\text { lud mental sería más asesoramiento para su } \\
\text { prevención }\end{array}$ & & & & & \\
\hline $\begin{array}{l}\text { 12. Considero que reflexionar sobre la Salud Labo- } \\
\text { ral en nuestro centro ha sido muy conveniente }\end{array}$ & & & & & \\
\hline
\end{tabular}

* El número 1 corresponde a la menor puntuación y el 5 a la mayor puntuación. 


\section{PRINCIPALES PROBLEMAS QUE AFECTAN AL PERSONAL DE LA EOI:}

1. Ordene estos problemas de salud física causados por su labor como personal en la EOI por orden de frecuencia en su vida.

\begin{tabular}{|l|l|}
\hline Resfriados & \\
\hline Gastroenteritis & \\
\hline Problemas de visión & \\
\hline Sobrecargas musculares & \\
\hline Trastornos en la voz & \\
\hline
\end{tabular}

\begin{tabular}{|l|l|}
\hline Problemas posturales & \\
\hline Dolores de cabeza & \\
\hline Dolores de garganta & \\
\hline Problemas alérgicos & \\
\hline Problemas de tensión arterial & \\
\hline
\end{tabular}

2. Posibles soluciones para los problemas de salud física más frecuentes:

3. Ordene estos problemas de salud mental causados por su labor como personal en la EOI por orden de frecuencia en su vida.

\begin{tabular}{|l|l|}
\hline Estrés & \\
\hline Ansiedad & \\
\hline Nerviosismo & \\
\hline $\begin{array}{l}\text { Angustia debido a } \\
\text { inseguridad laboral }\end{array}$ & \\
\hline Depresión & \\
\hline
\end{tabular}

\begin{tabular}{|l|l|}
\hline Falta de autoestima & \\
\hline Frustración & \\
\hline Insomnio & \\
\hline $\begin{array}{l}\text { Presión debido a exceso de } \\
\text { responsabilidad }\end{array}$ & \\
\hline Desestabilización emocional & \\
\hline
\end{tabular}

4. Posibles soluciones para los problemas de salud mental más frecuentes:

5. Observaciones: 